\title{
Análisis del discurso de los estudiantes de Magisterio sobre la contribución del practicum al desarrollo de su identidad profesional docente
}

\section{Analysis of the discourse of trainee teachers regarding the incidence of their practicum on the construction of their professional identity}

\author{
Miguel Pérez Ferra ${ }^{1}$ \\ mperez@ujaen.es \\ Rocío Quijano López \\ rquijano@ujaen.es \\ Universidad de Jaén, España
}

\section{Resumen:}

El estudio responde al análisis del discurso de los estudiantes de Magisterio sobre la incidencia del practicum en la construcción de su identidad profesional docente. Se partió de setenta y seis estudiantes, que han cursado el practicum de cuarto curso en 2016-17, Educación Primaria, pertenecientes a la Universidad de Jaén, seleccionando veintiuna narrativas, en función de la calidad de los relatos. Se han utilizado dos metodologías complementarias: el Análisis crítico del discurso y la Teoría fundamentada. Las narrativas de los estudiantes evidencian un discurso en torno a cuatro grandes categorías: "profesionalización docente", "uso de metodologías", "sentido ético" y "vocación", ámbitos que se manifiestan en un discurso diferenciado; bien en torno a un sentido racional de

\begin{abstract}
:
This study aimed to analyze the discourse of trainee teachers regarding the incidence of their practicums on the construction of their professional identity. The study surveyed seventy-six students all of whom did the fourth-year Primary Education practicum at the University of Jaén during academic year 2016-17. Twenty-one narratives were selected depending on the quality of the stories. Two complementary methodologies were used: Critical Discourse Analysis and Fundamental Theory. The students' narratives revolved around four major categories: "teacher professionalization", "use of methodologies", "ethical sense" and "vocation". These categories were characterized by implementing either a rational sense of conceiving teaching or a merely humanistic perception.
\end{abstract}

1 Dirección para correspondencia (correspondence address):

Miguel Pérez Ferra. Dpto. de Pedagogía. Universidad de Jaén. Facultad Humanidades y Ciencias de la Educación. Campus de Las Lagunillas. Edif. Humanidades y Ciencias de la Educación I (D2-017). 23071 Jaén (España). 
Análisis del discurso de los estudiantes de Magisterio sobre la contribución del practicum al desarrollo de su identidad profesional docente

Miguel Pérez Ferra y Rocío Quijano lópez

concebir la enseñanza; bien desde una percepción meramente humanística. En la propuesta de cambio se alude a la necesidad que tiene la Universidad de gestionar lo nuevo desde la tradición.

\section{Palabras clave:}

Análisis del discurso; alumnos de practicum; identidad profesional docente.
In the proposal for change made by the authors it was highlighted that the University should manage the new from the traditional.

\section{Key words:}

Speech analysis; practicum students; teachers' professional identity.

\section{Résumé:}

L'étude traite l'analyse du discours des étudiants de formation des enseignants du primaire sur l'incidence qu'a eu leur stage dans la construction de leur identité professionnelle en tant qu'enseignants. L'étude a commencé à partir de soixante-seize étudiants, qui ont fait le stage de quatrième année en 2016-17, d'enseignement primaire, qui font partie de I'Université de Jaén, et desquels on a sélectionné vingt et un récits, en fonction de la qualité des histoires. Deux méthodologies complémentaires ont été utilisées : l'analyse critique du discours et la théorie fondamentale. Les récits des étudiants présentent un discours autour de quatre grandes catégories : «professionnalisation des enseignants», «utilisation des méthodologies», "sens éthique» et «vocation», domaines qui se manifestent dans un discours différencié; aussi bien autour d'un sens rationnel de concevoir l'enseignement; que d'une perception simplement humaniste. Dans la proposition de changement, on fait référence à la nécessité de l'Université de gérer le nouveau depuis la tradition.

\section{Mots clés:}

Analyse du discours; élèves en stage; identité professionnelle; vocation éthique; méthodologies; professionnalisation.

Fecha de recepción: 15-2-2018

Fecha de aceptación: 14-5-2018

\section{Introducción y marco teórico}

Es preocupación creciente entre el profesorado definir "cómo" enseñar, interés menos acentuado en el "qué" y "para qué" de su actividad formativa, tal vez, porque en la base formativa de no pocos docentes no se ha contemplado la "integridad" del ser humano como definidora de la "integralidad" de su persona. Las razones afectan al profesorado y a los centros, pero transcienden su realidad. En este sentido, argumenta Escudero (2012):

Sería ingenuo, precisamente en estos tiempos, adoptar exclusivamente miradas didácticas u organizativas; es inexcusable convocar otras de mayor alcance. A pesar de su carácter personal y situado, nunca fue la innovación un fenómeno independiente de los contextos y tensiones sociales, políticas e ideológicas. (p. 6) 
Dos razones determinan la causa y el efecto de esta realidad, que afecta al ámbito de la educación; una, de carácter mediato, debida a la interpretación filosófica que se dio en su momento a uno de los grandes legados de la Modernidad, "el principio de acción"; otra, de carácter inmediato, consecuencia de la anterior, manifestada en trasmitir lo que se puede verificar experimentalmente, mitigando lo que transciende a lo meramente operativo.

El principio de acción subvierte su significado cuando se define como movimiento, cambio o producción, que sitúa a la persona como actor y elude su condición de autor (Foucault, 1990; Tell, 2014) de una acción no temática sino operativa (Llano, 1990). Sustenta esta nueva realidad la sustitución del concepto de "homo rationale" por el de "animal liberum", adoptada por Kant de Rousseau, que se prolonga hasta nuestros días e implica que la "acción humana" obedece a razones de "conciencia personal" (Ortega y Mínguez, 2005, p. 863). La acción educativa pierde su sentido moral y ético, quedando reemplazados por un mero compromiso de buena voluntad, sustentado en el respeto al deber. Como ha afirmado Mínguez (2010) esta situación:

(...) ha provocado una tendencia a valorar más lo útil, a transmitir lo que se puede verificar de modo experimental y a equiparar lo verdadero con lo numérico, lo positivo o lo empíricamente contrastable. (p. 48)

Lo descrito ha influido en los planes de estudio de los títulos de Maestro; en la formación del profesorado universitario de la titulación; en las dinámicas que se desarrollan en las aulas no universitarias y en la legislación vigente, realidades que condicionan el discurso de los estudiantes del practicum sobre qué es ser docente, hacia una percepción neopositivista.

En los nuevos planes de estudio se han incorporado, acertadamente, métodos activos de trabajo, que solo han definido la "accion" como finalidad en sí misma, obviando la propia naturaleza del conocimiento y su función en el desarrollo y desempeño de la persona (Monereo y Pozo, 2003), reduciendo, erróneamente, el concepto de sociedad del conocimiento a la disponibilidad de información, obviando su verdadera realidad: generar conocimiento desde la información disponible, entendida como praxis personal.

Pérez-Ferra, Quijano y Ocaña (2013) se han referido a esta realidad, indicando que: 
Análisis del discurso de los estudiantes de Magisterio sobre la contribución del practicum al desarrollo de su identidad profesional docente Miguel Pérez Ferra y Rocío Quijano lópez

El saber va más allá de la información, supone un crecimiento interno que se manifiesta en la excelencia de la capacidad operativa, admite una elaboración personal que genera conocimiento interno, (...) supone el enriquecimiento de nuestro ser práctico y el incremento de nuestra capacidad de obrar (p.242)

La formación del profesorado de la titulación también incide en el discurso de los alumnos sobre la identidad profesional docente, en adelante IPD. Los docentes noveles no solo deben conocer la ciencia que cultivan y su realidad epistemológica, sino también el sentido ético que define las relaciones entre profesores y estudiantes (Bolívar, 2017), que transciende la instrucción y contempla el apoyo y la orientación. Pero la acentuada orientación de la Universidad al ámbito empresarial ha supuesto obviar parte de los valores que los universitarios adquirían, quedando la formación reducida a lo instrumental.

La percepción de los tutores del practicum sobre "qué", "cómo" y "para qué enseñar", también incide en la definición de la IPD por los estudiantes. A veces, las concepciones de los tutores surgen del desencuentro entre las formulaciones de la ciencia didáctica, las apreciaciones de la legislación educativa vigente y, en los momentos actuales, de la percepción reduccionista de la secuencia curricular (Díaz-Barriga, 2013).

En el ámbito legislativo, la LOMCE también ha propiciado un discurso basado en la acción, orientado al predominio de la calidad educativa como selección competitiva, en detrimento de la equidad (Bayona-Aznar, 2013). Es un discurso en el que prevalece la "utilidad" sobre el "valor". Sin obviar la dimensión positiva de los aprendizajes que se adquieran y se puedan verificar experimentalmente, lo negativo es que la finalidad de lo legislado sea reducir el discurso a una "cronolatría" de la acción verificable, para desarrollar personas competitivas, que no competentes.

Definidos los principios argumentativos generadores de la realidad descrita y los canales de difusión en la escuela, parece oportuno especificar qué estructura presenta el discurso de los estudiantes de practicum sobre el desarrollo de la IPD.

El discurso evidencia dos percepciones claramente definidas sobre la construcción de la IPD, recogidas en la fundamentación de esta colaboración; una basada en "la conciencia personal", cuyo referente es la misma persona, que asume su quehacer docente como mero compromi- 
so de buena voluntad, razones que sustentan el discurso de la mayoría de los estudiantes del practicum; otra, definida por el compromiso moral, que supone hacerse cargo del alumnado, verdadera razón del desarrollo profesional. Ambas concepciones se definen a partir de procesos de socialización, determinantes en la concepción que cada maestro/a tiene de sí mismo sobre la construcción de su IPD (Bolívar, Domingo y PérezGarcía, 2014; Jarauta, 2014).

La concepción de los estudiantes que han realizado el practicum sobre el desarrollo de la IPD, queda definida de modo más relevante por 4 categorías: desarrollo profesional, uso de metodologías, compromiso ético y vocación profesional.

Respecto al desarrollo profesional, gran parte de los estudiantes comparten la primacía otorgada a las metodologías activas que, siendo necesarias, también se deben completar con "una orientación ético-moral sobre los criterios que deberían regular la vida humana y las relaciones personales" (Mínguez, 2010, p. 47). Hay investigaciones que evidencian un discurso en los estudiantes del practicum en el que la educación asume, pero transciende lo meramente instrumental, situando los estudiantes la centralidad teleológica de la formación en el reconocimiento y formación de la integridad del alumnado (Rojas y Ballester Pérez, 2017).

Las metodologías son fundamentales en la formación del profesor. Es importante el "paso de profesor experto en los contenidos de la disciplina a experto en la didáctica de la disciplina" (Zabalza-Beraza y ZabalzaCerdeiriña, 2012, p. 100), lo es en cuanto verifica la experticidad en la materia, el conocimiento epistemológico y gestiona los procedimientos más adecuados para generar aprendizajes. Los alumnos del practicum vinculan el desarrollo de la IPD con estas tres categorías, que se retroalimentan e infieren entre ellas.

El compromiso ético constituye un "nudo gordiano" en el que la responsabilidad personal que suscita, lo define como el compromiso basado en creencias pedagógicas, para hacerse cargo del "otro", para desarrollarlo en toda su "integralidad".

La vocación profesional se vincula con el sentido ético de la educación e incide en el desarrollo de la IPD, determinado por la pasión que enfatiza la necesidad de autoconocimiento de los valores del docente, constitutivos del impulso para mejorar y retroalimentar la autoestima a partir de los logros alcanzados (Day, 2006). En la vocación se sustenta el deseo y compromiso de acogida, al que urge la ética. Este discurso 
suele ser el de los estudiantes que han consolidado creencias basadas en sus gratos recuerdos de escolarización, que han sido determinantes para la elección de los estudios de Magisterio (Serrano-Rodríguez y Ponte, 2016). Otras concepciones vinculan el sentido ético, la profesionalización y la vocación a un cumplimiento del deber, basado en la buena voluntad.

\section{Relato metodológico}

\section{Objetivos de la investigación}

- Determinar y analizar la estructura del discurso de los estudiantes de Magisterio, respecto a la construcción de su IPD, a partir del practicum.

- Reconocer las claves filosóficas que inciden en el pensamiento de los estudiantes y definen su concepción epistemológica de la Didáctica y el Currículum.

- Identificar los agentes educativos que inciden en la orientación de la construcción de la IPD de los estudiantes de Magisterio. Hipótesis de investigación

\section{Metodología}

Se parte del análisis de narrativas de los estudiantes sobre su experiencia relativa al practicum, a fin de conocer qué creencias informan su discurso sobre la construcción de la IPD.

El estudio recoge las narrativas de estudiantes que realizan el practicum en tercer año de carrera, lo que supone el primer contacto con el alumnado. Hay que considerar que un gran porcentaje de estos alumnos son la primera generación universitaria de sus respectivas familiar, siendo la Universidad de Jaén la institución universitaria española con mayor porcentaje de estudiantes en esta situación. Para la elaboración de las narrativas se realizó un taller con los estudiantes, indicándoles que debían reflejar su percepción sobre qué es ser maestro, no por lo que habían estudiado, sino por su percepción personal, así como qué aspectos abarca esa realidad profesional.

La investigación se enmarca dentro de un proyecto más amplio al- 
cance que pretende abordar cómo se construye la identidad profesional docente, partiendo de los estudios de Educación Primaria de los estudiantes y concluyendo con los 5 primeros años de ejercicio profesional. En cuanto a la recogida de las narrativas, se realizó en el último mes que los estudiantes estuvieron en prácticas, siendo voluntaria la realización, y no figurando ninguna identificación personal.

Se ha planteado un estudio cualitativo porque: "La investigación cualitativa requiere una metodología sensible a las diferencias, a los procesos singulares y anómalos, a los acontecimientos y a los significados latentes" (Tejedor, 1988, p. 88).

El análisis de la información facilitada por los informantes se ha Ilevado a cabo mediante dos metodologías complementarias; en primer lugar, mediante el análisis crítico del discurso (Van Dijk, 2003), para identificar y comprender las "macroestructuras semánticas" que ha generado el discurso; en segundo lugar, mediante la Teoría Fundamentada "Grounded theory", que ha permitido la emergencia de teoría inductiva ((Hernández-Carrera, 2014; San Martín, 2014; Trinidad, Carrero y Soriano, 2006).

Las dos metodologías de referencia definen el marco metodológico para analizar y generar teoría desde la información que aporta el discurso, respectivamente (Domingo y Martos, 2016).

\section{Determinación de los casos objeto de estudio, procedimiento y recogida de análisis}

\section{Fase 1: Determinación de los Casos Objeto de eStudio y DiseÑo del ANÁlisis DE LA INFORMACIÓN}

La población la integran setenta y seis estudiantes, que han cursado el practicum de cuarto curso en 2016-17, Educación Primaria, pertenecientes a la Universidad de Jaén, seleccionando veintiuna de las narrativas aportadas, que fueron cuarenta y siete, en función de la calidad de los relatos.

Se ha realizado un diseño de relatos cruzados entre las categorías generadas mediante un proceso inductivo que ha partido del análisis de las narrativas de los estudiantes sobre la concepción que tienen de la función docente y la incidencia del practicun en la construcción de su IPD (Day, 2006; Vezub, 2007). 


\section{FASE 2: DiSEÑO Y ANÁLISIS DE LA INFORMACIÓN}

A partir de la configuración metodológica definida, se han analizado las narrativas de los estudiantes. En una primera fase, dedicada a la estructura del discurso, se han identificado los temas (categorías) más significativos, categorizándolos mediante un orden de frecuencias, que determinó la idea central del discurso y las subordinadas, que definen las temáticas del mismo, proceso que se realizó con el programa NVivo11-Plus.

En un segundo momento, se han identificado las temáticas que articulan las narrativas de los estudiantes y su orientación, analizando el uso de las palabras, la estructura proposicional, la coherencia del discurso y la generación de posibles inferencias lógicas en las narraciones (Bocaz, 1993). A partir de aquí, y mediante el método de comparación constante se ha realizado la codificación teórica, generando categorías mediante un proceso inductivo, a fin de establecer los conceptos centrales que permitieran la interpretación del discurso de los estudiantes sobre la percepción de la IPD, después de su experiencia en el practicum (Marín, Hernández y Flores, 2016).

\section{Caracterización del discurso de los estudiantes}

El discurso de los informantes quedó definido por el predominio del "desarrollo profesional docente" como definidor de la IPD. La frecuencia de palabras, tabla 1 y el gráfico de redes, figura 2, definen un discurso centrado en el alumnado, con la acción desarrollada en el tiempo de clase y cuya razón de ser es el aprendizaje. El profesor es un facilitador de los procesos instructivos en el contexto de aula y centro, discurso en el que predomina la instrucción como finalidad última del proceso formativo.

Las narrativas aluden al replanteamiento metodológico, tanto en la Universidad como en Primaria, orientado hacia el uso de metodologías activas, que favorecen el aprendizaje autónomo y el desarrollo de competencias (Palazón-Pérez de los Cobos, Gómez-Gallego, GómezGallego, Pérez-Cárceles y Gómez-García, 2011; Robledo, Fidalgo, Arias y Álvarez, 2015). Pero el discurso no recoge las dimensiones formativas de las competencias, ya que se contempla el "saber" y "saber hacer" mayoritariamente y se elude "el saber ser y" "saber estar"; es decir, la dimensión formativa. 
Tabla 1. Sustantivos vinculados al campo semántico del desarrollo de la identidad profesional mediante el practicum.

\begin{tabular}{lccl}
\hline Palabra & Conteo & $\begin{array}{c}\text { Porcentaje ponderado } \\
(\%)\end{array}$ & Palabras similares \\
\hline Alumnos & 790 & 1,84 & Alumnos, alumnado, niños. \\
\hline Clase & 265 & 0,59 & Clases \\
\hline Aprendizaje & 234 & 0,56 & Aprendizajes \\
\hline Profesores & 215 & 0,42 & Profesorado \\
\hline Conocimiento & 207 & 0,40 & Conocedores \\
\hline Resolutivo & 203 & 0,38 & Resolutivos \\
\hline Aula & 200 & 0,35 & Aulas \\
\hline Centro & 162 & 0,38 & Centros \\
\hline Trabajo & 136 & 0,32 & Trabajar \\
\hline Prácticas & 122 & 0,29 & Práctica \\
\hline Actividades & 105 & 0,25 & Actividad \\
\hline Metodología & 103 & 0,25 & Metodologías \\
\hline
\end{tabular}

Fuente: Elaboración propia.

\section{Estructura del discurso de los estudiantes en torno al desarrollo de la identidad profesional}

A partir de los criterios definidos, los nodos categoriales quedan estructurados según las frecuencias que reflejan las interacciones entre los mismos, tabla $\mathrm{n}^{\circ} 2$, figura 2 .

Después de la experiencia del practicum, los estudiantes definen la IPD vinculada al "desarrollo profesional", considerado como conocimiento y puesta en práctica de "metodologías activas". El "compromiso ético" tiene vinculación con "desarrollo profesional", pero entendida esa ética, básicamente, como deber cumplido. La vocación se mueve en una dialéctica, que se entiende como sentido profesional del deber, desde una perspectiva kantiana, o bien como retroalimentación del trabajo bien hecho, que influye en el rendimiento y satisfacción del docente. Pero habrá que profundizar en el análisis de las categorías descritas, para determinar qué entienden los estudiantes por esos términos, ya que los discursos difieren en sus apreciaciones. 
Análisis del discurso de los estudiantes de Magisterio sobre la contribución del practicum al desarrollo de su identidad profesional docente

Miguel Pérez Ferra y Rocío Quijano López

Tabla 2. Matriz de codificación, sobre frecuencias cruzadas entre los nodos temáticos.

\begin{tabular}{lcccc}
\hline & $\begin{array}{c}\text { Compromiso } \\
\text { ético }\end{array}$ & $\begin{array}{c}\text { Desarrollo } \\
\text { profesional }\end{array}$ & $\begin{array}{c}\text { Uso de } \\
\text { metodologías }\end{array}$ & $\begin{array}{c}\text { Vocación } \\
\text { profesional }\end{array}$ \\
\hline Compromiso ético & 54 & 32 & 16 & 21 \\
\hline Desarrollo profesional & 32 & 86 & 36 & 27 \\
\hline Uso de metodologías & 16 & 36 & 58 & 7 \\
\hline Vocación profesional & 21 & 27 & 7 & 46 \\
\hline
\end{tabular}

Fuente: Elaboración propia.

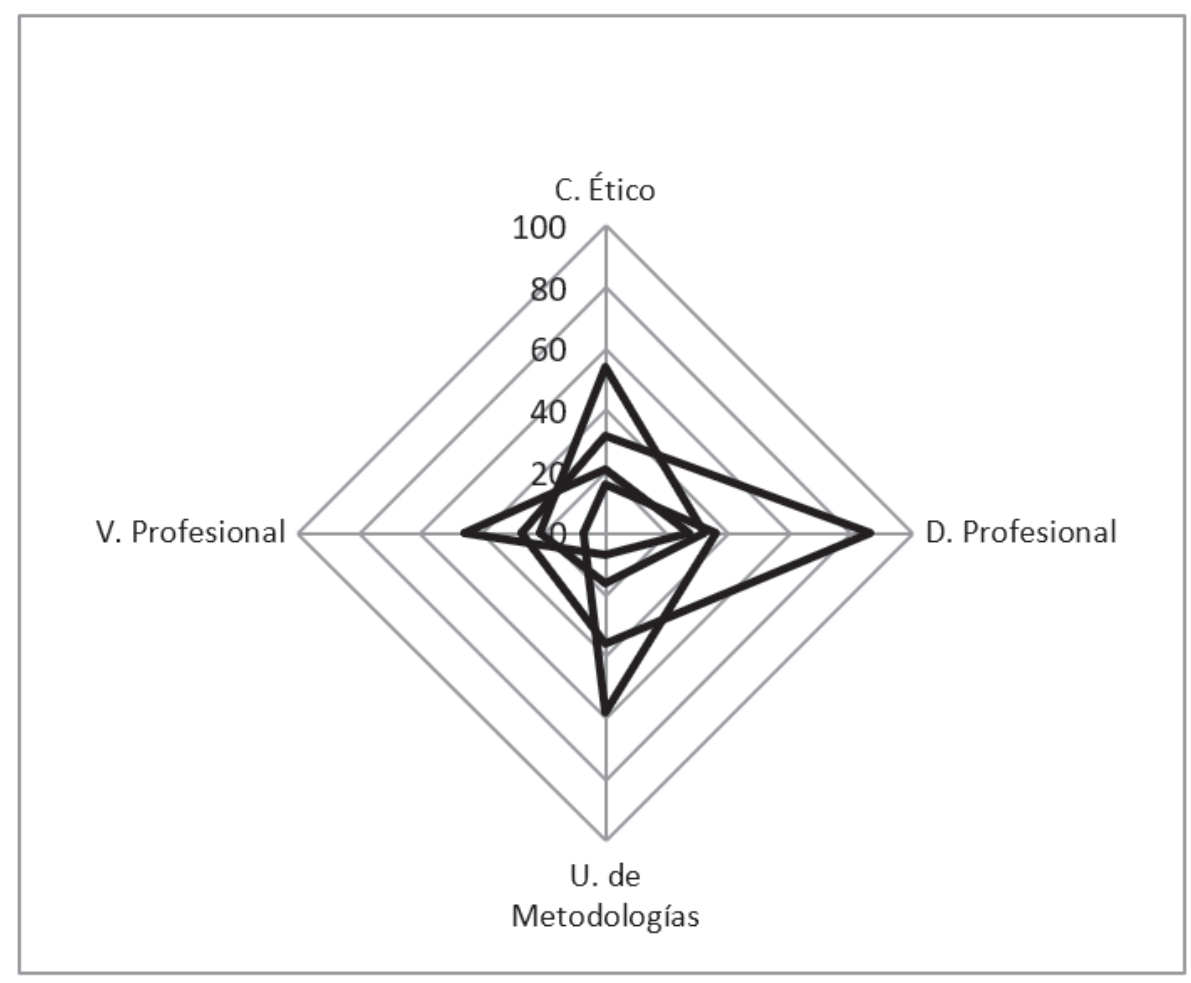

Figura 1. Gráfico de redes sobre frecuencias cruzadas entre categorías temáticas.

Fuente: Elaboración propia. 


\section{Discurso de los estudiantes sobre la contribución del practicum a la definición de su identidad profesional}

\section{El discurso de los estudiantes sobre el "desarrollo profesional"}

Las narrativas consideran "el desarrollo profesional" como eje vertebrador de la IPD de los estudiantes, concretamente ochenta y seis referencias se aportan sobre la mencionada categoría.

La utilización de términos como: "evolucionar", "vivir la experiencia", "estar con el profesorado", "observar la manera de trabajar", "analizas", "estrategias que emplean", evidencian que el estudiante se posiciona de modo expectante y con deseos de incorporar las vivencias u observaciones para crecer en su desarrollo profesional.

Se pone de manifiesto en las narrativas, que bastantes estudiantes solo observan la acción del profesorado, sin participación activa. Inciden en que quieren formarse en el conocimiento de métodos activos para que los niños adquieran aprendizajes y desarrollen su capacidad para resolver situaciones concretas. Sin embargo, no hay una implicación que favorezca el desarrollo del "ser" y "saber estar" de los niños.

Referencia 1 - Cobertura 17,95\%

"Durante estos dos meses que he realizado de prácticas he evolucionado a un nivel muy bueno. El estar unos meses con los alumnos y vivir la experiencia de ser docente, de por sí hace que evoluciones como profesor, que te formes profesionalmente. Posteriormente, el estar día a día con tu tutor y los demás profesores de las diferentes asignaturas, hace que observes la manera de trabajar que tienen cada uno y las estrategias que emplean para llevar a cabo el proceso enseñanza-aprendizaje. Analizas cómo lo llevarías a cabo tú y en que podrías mejorar en algunos aspectos que no creías que podían darse así. Se trata de que los niños adquieran aprendizajes y desarrollen su capacidad resolutiva".

Otro grupo de estudiantes menos numeroso, considera el desarrollo profesional como resultado de un trabajo compartido, cuya finalidad es la formación de la "integralidad" de los estudiantes, que asume su instrucción, pero la transciende. Valoran la formación del alumnado como un fin común compartido por el profesorado. Son estudiantes que han realizado 
Análisis del discurso de los estudiantes de Magisterio sobre la contribución del practicum al desarrollo de su identidad profesional docente Miguel Pérez Ferra y Rocío Quijano López

el periodo de practicum en centros más participativos, cuyos tutores vinculan la enseñanza no solo a una acción social, sino que se implican en su totalidad con la generación de nuevos proyectos de vida (Ibáñez-Martín, 2017). Los informantes que así opinan, suelen ser personas comprometidas con la formación en ámbitos informales: Movimiento Scouts, etc.

Referencia 2 - Cobertura 1,85\%

"A fin de cuentas, lo que importa es poder trabajar por un fin común, el desarrollo personal de los alumnos y olvidar las diferencias personales por el bien de los alumnos, en quienes repercuten nuestros actos. He tenido libertad para trabajar con mi tutor y el resto de profesores, que es como verdaderamente se aprende. Debo decir que mi trabajo como voluntario en las salidas que organizan los $\mathrm{HH}$. Salesianos me ha sido de gran utilidad para completar mi formación en el practicum".

\section{Discurso de los estudiantes sobre el "uso de metodologías"}

Esta categoría supone el principal elemento de acción que, según la estructura del discurso, sustenta el desarrollo de la IPD (profesionalización). Se referencia en cincuenta y ocho ocasiones y se vinculan al "desarrollo profesional" en treinta y seis, aunque hay una baja relación con el "compromiso ético" y la "vocación profesional".

Los informantes contraponen metodología tradicional y metodología activa, aunque en esta ocasión, aportando una visión más formativa, ya que se tiene en cuenta la singularidad del estudiante y la incidencia de los procesos de enseñanza-aprendizaje en su socialización, vinculando los métodos a principios activos.

Referencia 3 - Cobertura 7,97\%

“(...) he podido darme cuenta de aspectos metodológicos y pedagógicos que en un futuro me gustaría utilizar en un aula. Son metodologías activas que permiten al niño desarrollar procesos de autoaprendizaje. Pero la formación constante de los docentes es imprescindible para trabajar de este modo.

Se ha trabajado mediante proyectos, ya que permiten que los alumnos pongan en práctica sus conocimientos de manera autónoma, y que se involucren en su propio aprendizaje, construyéndolo de manera activa." 
Otros informantes profundizan algo más en la percepción que tienen sobre las metodologías, vinculándolas al interés por el desarrollo de procesos que ejercitan capacidades cognitivas, pero son solo capacidades cognitivas, en ningún momento abordan las afectivas, las relacionadas con la interacción social o las capacidades psicomotoras. Se aprecia, por tanto, en la formación previa de los estudiantes el desconocimiento de "la 'integridad' del ser humano (que) determina la 'integralidad' de los elementos constitutivos de la persona" (Pérez-Ferra, 1993, p. 17). Se evidencia, en cierto modo, que el concepto de acción ha "calado" en los estudiantes con un sentido finalista.

\section{Referencia 4 - Cobertura 2,74\%}

"Las propuestas de aprendizaje desarrollan variedad de procesos cognitivos. El alumnado es capaz de poner en práctica un amplio repertorio de procesos, tales como: identificar, analizar, reconocer, asociar, reflexionar, razonar, deducir, inducir, explicar, etc."

En otras narrativas emergen fragmentos en los que se muestra interés por personalizar la enseñanza, aportando intencionalidad y sentido de finalidad a los métodos, calificando su acción como didáctica, pero también como formativa. Siguen construyendo este tipo de discurso los estudiantes vinculados a instituciones o movimientos comprometidos con la formación no formal de los niños: No se trata simplemente de que los alumnos aprendan a hacer, sino que la acción esté orientada hacia una realidad finalista, siempre en consonancia con lo que supone ser "persona".

Referencia 5 - Cobertura 6,36\%

“(...) he aprendido a utilizar métodos que tienen en cuenta los diferentes ritmos de aprendizaje del alumnado, favoreciendo la capacidad de aprender por sí mismos, promover el trabajo en equipo y socializarse con los demás".

Me refiero a los proyectos, pero no entendidos solo como metodología activa para aprender, sino orientándolos como procedimientos de acción para que lo aprendido por los alumnos sea útil para que aprendan a ser colaborativos, desarrollen la inteligencia emocional y permitan que los conocimientos adquiridos faciliten a los alumnos su formación en valores. No 
Análisis del discurso de los estudiantes de Magisterio sobre la contribución del practicum al desarrollo de su identidad profesional docente

Miguel Pérez Ferra y Rocío Quijano López

veo las metodologías activas como un fin en sí mismas, sino como punto de partida para la formación integral de los estudiantes".

\section{El discurso de los estudiantes sobre el "compromiso ético" del docente}

La tercera categoría, en nivel de incidencia sobre el "desarrollo profesional" es "compromiso ético", referenciado por los informantes en cincuenta y cuatro ocasiones, y vinculado al "desarrollo profesional" en treinta y dos. Sin embargo, su relación con "vocación profesional", veintiuna frecuencias y dieciséis con el "uso de metodologías", posiciona el "compromiso ético" a cierta distancia de la formación didáctico-curricular y de la "vocación profesional", entendido como aspecto que mueve hacia el compromiso.

La mayoría de informantes centran el discurso sobre esta categoría en tres términos: "implicación", "trabajo" e "interés por seguir aprendiendo", discurso que define un panorama en el que la dimensión ética es considerada como aspecto complementario de la acción educativa (Mèlich y Boixader, 2010; Mínguez, 2010); es decir, se entiende como un proceso de socialización del código moral sancionado por la sociedad.

Los estudiantes consideran la necesidad de comprometerse con la formación continuada como docentes, pero entendida como la "actualización didáctica" que demandan las vanguardias sociales. Ello constituye el compromiso central y medio más adecuado para llevar a cabo su actividad profesional. Ese compromiso es más social que ético, en el sentido de respeto a un código ético de la profesión, pero disociando sociedad y ética. Es el resultado "de una crisis que pone de manifiesto las carencias de espíritu cívico, que denotan la falta de un marco ético (...), capaz de estimular la responsabilidad social y un buen uso de la libertad" (Camps, Cortina y García-Delgado, 2012).

Referencia 6 - Cobertura 6,59\%

“(...) la enseñanza parece fácil pero no lo es, se trata de tener un sentido de responsabilidad para hacer llegar a los alumnos los conocimientos que han de adquirir. Para ser maestro se necesita tener asumidos una serie de conocimientos para poder transmitirlos al alumnado.

La ética es uno de los puntos básicos para enseñar en la escuela, ya 
que suponen conocer y cumplir con el currículum que establece, es nuestro caso, la Consejería de Educación de la Junta de Andalucía".

Pero también hay reflexiones más profundas en el discurso, que surgen de un compromiso radical con el alumno, basado en el hecho de "hacerse cargo de él" (Ortega-Ruiz, 2004, p. 5), siendo el practicum la ocasión para poner a los estudiantes de Magisterio ante un modo de concebir la educación, vinculando "integridad" e "integralidad" en la formación del alumnado. Son estudiantes que, además de compartir aula con tutores implicados en la formación, llevan a cabo actividades asistenciales y consideran el sentido ético como intrínsecamente unido a la relación educativa. No hay "compromiso ético" si la educación no comporta hacerse responsable del "otro".

Referencia 7 - Cobertura 3,62\%

"Esta experiencia ha supuesto un aumento de las ganas de luchar por la educación. Cuando estás en la Universidad estudiando las diferentes asignaturas, no te das cuenta realmente de lo que supone enseñar a un grupo de alumnos los conocimientos que necesitan. Y no sólo eso, sino que también se enseñan valores, actitudes y formas de afrontar la vida. Personalmente, considero este aspecto muy importante, ya que los alumnos cuando salen del colegio deben saber respetar a los demás, siempre con la acción conjunta de las familias.

He tenido la oportunidad de comprobar que mi participación en el proceso formativo se ha iniciado con una mutua adaptación y reconocimiento entre los niños y yo. Es ahí donde han valorado que son alguien para el profesor, reconocidos en su singularidad personal. Es por ello, que aquí he aprendido que hablar de educación es evocar una experiencia irrepetible, singular, donde la ética profesional me urgen a "hacerme cargo" de cada niño, con sus particularidades".

\section{El discurso de los estudiantes sobre la "vocación profesional"}

La cuarta categoría tiene cuarenta y seis frecuencias, siendo su incidencia compartida con el "desarrollo profesional" de veintisiete frecuencias, la menor de todas las categorías, incidencia que también disminuye en su relación con el "compromiso ético", veintiuna frecuencias, ya que son mayoría los informantes que perciben una realidad profesional en la 
Análisis del discurso de los estudiantes de Magisterio sobre la contribución del practicum al desarrollo de su identidad profesional docente Miguel Pérez Ferra y Rocío Quijano López

que el profesor es solo un profesional con una función social, disociada, cada vez más, de la "vocación profesional".

Esta categoría se considera como conjunto de valores que impulsan la mejora de la autoestima, consecuencia de los logros alcanzados con los alumnos y con uno mismo. Cobo-Suero (2013) indica que:

(...) cabe establecer diferencias entre el docente profesional y el docente vocacional en el modo de sentirse realizados. El primero se satisface con el deber cumplido, cuando consigue los fines de la actividad profesional, mientras que el segundo añadirá a lo anterior la satisfacción de haberse realizado profesionalmente (p.128).

Atendiendo a estas reflexiones previas, hay estudiantes que plantean en sus narrativas un sentido del trabajo concebido como deber cumplido y fines alcanzados, sin proyectar su actividad docente a la atención de la singularidad de los alumnos, entendiendo por ello el "compromiso ético". Son los docentes que cumplen con la profesionalidad.

Referencia 8 - Cobertura 12,42\%

"Durante todo este tiempo he aprendido que los maestros tenemos un compromiso; es un compromiso institucional con la Administración educativa, que se refleja en los niños, que son los destinatarios del currículum, cuyos contenidos han de aprender y evaluar según los estándares de evaluación.

También he visto reflejado en mi tutora la importancia y tranquilidad que da el deber cumplido, logrando la preparación necesaria para que los niños y niñas aprendan. También la importancia que tiene informar a las familiares de cómo van sus hijos o hijas. En definitiva, el cumplir los compromisos profesionales que requiere ser maestro o maestra".

Sin embargo, cuando las narrativas de los estudiantes se han orientado a describir que han descubierto su vocación profesional, el discurso cambia radicalmente, aunque estos alumnos son minoría, su percepción tiene en cuenta la profesionalidad, pero la transcienden, valorando el deber cumplido como punto de partida para realizarse profesionalmente; desarrollar su motivación y fortalecer las creencias pedagógicas que definen su identidad profesional docente. Es un discurso que considera que la escuela tiene cada día un nuevo afán. Suelen ser aquellos estu- 
diantes que vinculan vocación profesional a su fortalecimiento del proyecto ético de vida.

Referencia 9 - Cobertura 1,42\%

"He aprendido a tener más profunda y real conciencia de mi vida, el mundo en el que vivo, qué hago, qué puedo hacer, proporcionándome herramientas para gestionar mí día a día e interaccionar con un entorno en el que la norma es el cambio y la mejora de los alumnos y alumnas.

Otros informantes vinculan la "vocación profesional", en este caso, a contrastar cuando se ponen en contacto con la realidad del aula, si realmente son capaces de llevar a cabo la función docente. En un proceso para contrastar su autoestima y mejorar el autoconcepto, infiriendo una concepción de la educación emparentada con una actividad que implica responsabilidad social".

Referencia 10 - Cobertura 1,25\%

"También, me ha ayudado a confiar más en mi misma y a saber que puedo enfrentarme a distintas situaciones dentro del aula; descubriendo que esta profesión todos los días es distinta, en la que cada jornada te plantea un reto distinto: que tus alumnos aprendan y aprendan de la mejor forma siendo felices".

\section{Conclusiones}

El discurso de los estudiantes sobre la experiencia del practicum en el desarrollo de su IPD se estructura en torno a 4 categorías, que se referencian atendiendo a su significación en el discurso: "Desarrollo profesional", "uso de metodologías", "compromiso ético" y "vocación profesional".

La estructura del discurso de los estudiantes queda polarizado en torno a dos concepciones; una, basada en la orientación de su actividad docente, que obedece a razones de conciencia personal, cuyos referentes mediatos están en Kant, con quien "(...) el hombre se convierte en autolegislador, siendo la buena voluntad la que determina la bondad de las acciones del profesor" (Ortega y Mínguez, 2005,p. 863), o bien: "se entiende la educación moral como un proceso de socialización del 
código moral explícito, sancionado por la sociedad" (Ortega y Mínguez, 2004 , p. 864). Son alumnos que han recibido una formación inicial basada en la acción, la experimentalidad y en la verificación de las hipótesis que genera, sin transcendencia a otros ámbitos.

Un segundo grupo de estudiantes vincula el discurso sobre la incidencia del practicum en el desarrollo de la IPD a una profesionalización cuyo soporte lo constituye el "sentido ético" y la "vocación profesional", verdaderos referentes de una educación con rostro humano. No es una relación basada en la elección personal, ni profesional, sino en la verdadera realidad ontológica de la profesión docente, "hacerse cargo del otro (...) responder al 'otro' y del 'otro" (Mínguez, 2010, p. 47). Son alumnos que tienen "entrañas de compasión hasta hacer (suya) la situación del otro, hacerse cargo de él" (Ortega y Gárate, 2017, p. 26), cuya formación ha surgido en entornos asistenciales y de voluntariado y, también, en espacios reducidos de la formación inicial. Son estos ámbitos los que determinan la orientación del discurso, dominante entre los alumnos con una visión racionalista.

Los estudiantes que relacionan el "desarrollo profesional" exclusivamente al "uso de metodologías", han desarrollado sus creencias pedagógicas en entornos próximos al "mecanicismo cosmológico", que acontece cuando se pretende compaginar comprensión y explicación causal, sin dejar clarificada la articulación entre causalidad mecánica y causalidad final. La utilización de metodologías activas, siendo adecuadas para la formación del alumnado, no ha resuelto el problema secular del legado que nos dejó la Modernidad en la "acción", "el para qué", quedando reducida a mera cronolatría o reduciendo la persona a instrumento al servicio de la producción, neopositivismo.

Las creencias pedagógicas evidencian una percepción de la formación basada en la cosmovisión de lo metodológico, cuya aproximación actual atestigua que la explicación de la sociedad del conocimiento está ampliamente definida por el hecho educativo, ya que a partir de la segunda mitad del siglo XX, la educación deja de considerarse exclusivamente como espacio para el desarrollo personal y comienza a tener como referentes los objetivos sociales, al considerarse aspecto básico del desarrollo económico (Esteve, 2009, p. 18).

La aproximación a la construcción de la IPD considerada como optimización de la formación docente en el plano metodológico incide en el sentido ético, entendido más como mero compromiso social, que 
comporta una deontología, cuyos códigos éticos “(...) más cercanos se orientan a la descripción de una práctica social específica (...) que a una exigencia ética" (Ibáñez-Martín, 2017, p. 163), lo que acontece cuando lo social se disocia de lo moral.

Otros estudiantes, en menor número, abordan su formación profesional entendida como compromiso radical con el alumnado, que transciende la instrucción y abarca: "La 'integridad' del ser humano, determinante de la 'integralidad' de los elementos constitutivos de la persona" (Pérez-Ferra, 1993, p. 17).

La realidad descrita, en cuanto al modo de entender el compromiso, conduce a percibir los dos modos de percibir la vocación expresados por los estudiantes; de una parte, quienes entienden y viven la vocación como pulsión creciente que busca el servicio al "otro" y genera una relación cuya radicalidad estriba en el vínculo que se establece entre maestro y alumno, en una situación educativa que busca la ayuda al "otro" mediante la acogida y el compromiso, aspectos que potencian el interés por la profesión. Son estudiantes generalmente comprometidos con causas sociales o movimientos juveniles, en cuyas actividades han descubierto su vocación profesional, en el sentido genuino del término, sin obviar las vivencias surgidas junto a sus profesores; de otra parte, la de aquellos estudiantes, mayoría, que subvierten el sentido de la vocación, entendida como compromiso personal para actuar como profesionales; conociendo las exigencias de su tareas; disponiendo de conocimientos específicos y sentido de profesionalidad (Larrosa, 2010). Son aquellos estudiantes que han desarrollado sus creencias pedagógicas sobre la base de un discurso racional, fundamentado en los principios que sustentan la "Crítica de la Razón Pura" de Kant.

La estructura del discurso analizado plantea una serie de carencias debidas, fundamentalmente, a la formación inicial de parte del profesorado universitario, maestros, futuros maestros y responsables de la formación permanente.

La génesis del problema está en cuestionarse, como ha afirmado Llano (2012): sí “(...) una institución universitaria sabe cómo suscitar y gestionar lo nuevo" (p. 91). Para lograrlo, la Academia ha de procurar que el profesorado integre ser experto en los contenidos de la disciplina y ser experto en la didáctica de la disciplina; en caso contrario, el tránsito de la innovación a la ocurrencia será cuestión de segundos. 
Análisis del discurso de los estudiantes de Magisterio sobre la contribución del practicum al desarrollo de su identidad profesional docente

Miguel Pérez Ferra y Rocío Quijano lópez

\section{Referencias bibliográficas}

Bayona-Aznar, B. (2013). Los ejes de la LOMCE. Forum Aragón, 7, 3-5. Recuperado de http://feae.eu/doc/ara/revista_digital_forum_aragon_7.pdf

Bocaz, A. (1993). Generación de inferencias lógicas en la producción de discurso narrativo. Revista lenguas modernas, 20, 79 - 21.

Bolívar, A. (2017). Una ética profesional en la formación y relaciones universitarias. En M. Pérez-Ferra y J. Rodríguez-Pulido (Coords.). Buenas prácticas docentes del profesorado universitario (pp.15-29). Barcelona: Octaedro.

Bolívar, A.; Domingo, J. y Pérez-García, P. (2014). Crisis and Reconstruction of Teachers' Professional Identity: The Case of Secondary School Teachers in Spain. The Open Sports Science Journal, 7, 106-112. Doi: 10.2174/1875399X01407010106. Recuperado de https://benthamopen.com/contents/pdf/TOSSJ/TOSSJ-7-106.pdf

Camps, V.; Cortina, A y García-Delgado, J. L. (25 de septiembre de 2012).Democracia de calidad frente a la crisis. El País, p. 17.

Cobo-Suero, J. M. (2013). Ética profesional en ciencias humanas y sociales. Madrid: Huerga y Fierro.

Day, CH. (2006). Pasión por enseñar: La identidad personal y profesional del docente y sus valores. Madrid: Narcea.

Díaz-Barriga, A. (2013). Secuencias de aprendizaje. ¿Un problema del enfoque de competencias o un reencuentro con perspectivas didácticas? Profesorado. Revista de currículum y formación del profesorado.17(3), 11-33. Recuperado de http://www. redalyc.org/pdf/567/56729527002.pdf

Domingo, J. y Martos, J. (2016). Análisis del discurso político en España sobre el fracaso escolar en twitter. Archivos analíticos de políticas educativas, 40(70), 1 - 31. Recuperado de http://www.redalyc.org/pdf/2750/275043450070.pdf

Escudero, J. M. (2012). Claros y oscuros del Espacio Europeo de Educación Superior como innovación educativa, en C. Leite y M. Zabalza (Coords.) Ensino superior. Innovaçao e qualidade na docencia. CIIE. Recuperado de http://www.fpce.up.pt/ciie/cidu/index.html

Esteve, J. M. (2009). La profesión docente ante los desafíos de la sociedad del conocimiento. En C. Velaz de Medrano y D. Vaillant (Coordinadoras). Aprendizaje y desarrollo profesional docente (pp.17 - 57). Madrid: Fundación Santillana.

Foucault, M. (1990). ¿Qué es un autor? México: Universidad Autónoma de Tlaxcala.

Hernández-Carrera, R. (2014). La investigación cualitativa a través de entrevistas: su análisis mediante la teoría fundamentada. Cuestiones pedagógicas, 23, 187-210. Recuperado de: http://hdl.handle.net/11441/36261

Ibáñez-Martín, J. A. (2017). Horizontes para los educadores. Madrid: Dykinson.

Jarauta, B. (2014). La construcción de la identidad profesional del maestro de primaria durante su formación inicial. El caso de la Universidad de Barcelona. Profesorado. Revista de currículum y formación del profesorado, 21(1), 104-122. Recuperado de http://www.redalyc.org/html/567/56750681006/

Larrosa, F. (2010). Vocación docente versus profesión docente en las organizaciones. Educativas. REIFOP, 13(4), 43 - 51. Recuperado de http://www.aufop.com/aufop/ uploaded_files/articulos/1291992517.pdf 
Análisis del discurso de los estudiantes de Magisterio sobre la contribución del practicum al desarrollo de su identidad profesional docente

Miguel Pérez Ferra y Rocío Quijano López

Llano, A. (1990). Subjetividad moderna y acción trascendental. En R. Alvira, (coord.). Razón y libertad: homenaje a Antonio Millán-Puelles, (pp. 63-74). Madrid. España: Rialp.

Llano, A. (9 de noviembre de 2012). La Universidad y el tiempo [Mensaje en un blog]. Recuperado de http://joquivesa.blogspot.com.es/2011/11/la-universidad-y-el-tiempoalejandro.html

Marín, L. A.; Hernández, E. y Flores, Q. (2016). Metodología para el análisis de datos cualitativos en investigaciones orientadas al aprovechamiento de fuentes renovables de energía, Koinonia, 1(1), 1-17.

Mélich, J. C. y Boixader, A. (Coords.) (2010). Los márgenes de la moral. Una mirada ética a la educación. Barcelona, Graó.

Mínguez, R. (2010). La escuela hoy en la encrucijada. Hacia otra educación desde la ética de E. Lévinas. Teoría de la Educación, 22 (2), 43-61. Recuperado de https:// gredos.usal.es/jspui/bitstream/10366/121596/1/La_escuela_hoy_en_la_encrucijada_Hacia_o.pdf

Monereo, C. y Pozo, J.I. (Eds.) (2003): La universidad ante la nueva cultura educativa: enseñar y aprender para la autonomía. Madrid: Síntesis.

Ortega, P. y Mínguez, R. (2005). La educación moral, ayer y hoy. Revista galega do ensino, 13(46), 863-885. Recuperado de https://dialnet.unirioja.es/descarga/ articulo/2558629.pdf

Ortega-Ruiz, P. (2004). La educación moral como pedagogía de la alteridad. Revista española de pedagogía, 62(227), 5-30. Recuperado de http://www.jstor.org/ stable/23765747? seq=1\#page_scan_tab_contents

Ortega-Ruiz, P. y Gárate, A. (2017). Una escuela con rostro humano. Mexicali: México.

Palazón-Pérez de los Cobos, A.; Gómez-Gallego-Gallego, M.; Gómez-Gallego, J.; Pérez. Cárceles, Ma . C. y Gómez-García, J. (2011). Relación entre la aplicación de metodologías docentes activas y el aprendizaje del estudiante universitario. Bordón, 63(2), 27-40.

Pérez-Ferra, M. (1993). Actitudes positivas hacia el estudio. Estrategias de intervención para profesores de enseñanzas medias. Murcia: Obra Cultural-CajaMurcia.

Pérez-Ferra, M., Quijano, R. y Ocaña, Ma. T. (2013). El profesorado universitario ante el Espacio Europeo de Educación Superior: dos años después. Educatio Siglo XXI, 32(2), 235-254. Doi: 10.6018/j/308881.

Robledo, P.; Fidalgo, R.; Arias, O y Álvarez, M. L. (2015). Percepción de los estudiantes sobre el desarrollo de competencias a través de diferentes metodologías activas. Revista de investigación educativa, 33(2), 369-383. Doi: 10.6018/ie.33.2.201381.

Rojas, J. y Ballester-Pérez, B. (2017). Mancomunidad educativa y conciencia educadora: Hacia la formación de ciudadanía democrática. Sapienza organizacional, 4(7), 137 158. Recuperado de137 http://erevistas.saber.ula.ve/ index.php/ sapienza/article/ view/9341/9292

San Martín, D. (2014). Teoría fundamentada y Atlas.ti: recursos metodológicos para la investigación educativa. Revista electrónica de investigación educativa, 16(1), 104122. Recuperado de: http://www.scielo.org.mx/scielo.php?script=sci_arttext\&pid $=$ S1607-40412014000100008 
Análisis del discurso de los estudiantes de Magisterio sobre la contribución del practicum al desarrollo de su identidad profesional docente

Miguel Pérez Ferra y Rocío Quijano López

Serrano-Rodríguez, R. y Ponte, A. (2016). El desarrollo de la identidad profesional docente en la formación inicial del profesorado de secundaria. Enseñanza \& teaching, 34, 1-2016, pp. 35-55. Doi: 10.14201/et20163413555.

Tejedor, F. J. (1988). La estadística y los diferentes paradigmas en investigación cualitativa. Educar, 10, 79 - 101. Doi: 10.5565/rev/educar.463.

Tell, M. B. (2014). Después de la modernidad: reflexión sobre la acción de Dios, la acción del hombre, y cómo la razón se conoce en-acción a sí misma y lo que está encima de ella. Albertus magnus, 5(1), 11-32. Doi: 10.15332/s2011-9771.2014.0001.01.

Trinidad, A.; Carrero, V. y Soriano, R. M. (2006). Teoría fundamentada: La construcción de la teoría a través del análisis interpretacional. Madrid: Centro de Investigaciones Sociológicas.

Van Dijk, T.A. (2003). La multidisciplinariedad del análisis crítico del discurso: un alegato en favor de la diversidad. En R. Wodak y M. Meyer Coords.), Métodos de análisis crítico del discurso (pp.143-177). Barcelona: Gedisa.

Vezub, F. (2007). La formación y el desarrollo profesional docente frente a los nuevos desafíos de la escolaridad, Profesorado. Revista de currículum y formación del profesorado, 11(1), 1-23. Recuperado de http://digibug.ugr.es/bitstream/ 10481/17485/ 1/ rev111ART2.pdf

Zabalza-Beraza, M. A. y Zabalza-Cerdeiriña, Ma . A. (2012). Profesores y profesión docente. Entre el "ser" y el "estar". Madrid: Narcea. 